\title{
Conjugate natural convection heat transfer in
}

\section{an open-ended square cavity partially filled}

\section{with porous media}

\author{
Sheng Chen ${ }^{* 1}$, Wei Gong ${ }^{1}$,Yuying Yan ${ }^{1}$ \\ 1. Faculty of Engineering, The University of Nottingham, University Park, \\ Nottingham NGr 2RD, UK \\ * Corresponding author. Faculty of Engineering, University of Nottingham. E-mail \\ address: sheng.chen@nottingham.ac.uk
}

\begin{abstract}
Conjugate natural convection heat transfer in an open-ended square cavity, which is partially filled with porous media, is a useful research prototype to deepen our insight into many important practical applications, such as solar energy collectors. But surprising, until now there is no open literature on it. In addition, for traditional numerical approaches, it is a great challenge to model conjugate problems on fluid-porous interfaces. In the present work, firstly we develop a new lattice Boltzmann (LB) approach to overcome such difficulty. The present LB model is validated by three benchmark tests. With the aid of this LB approach, we investigate the effects of thickness of porous layer, fluid-to-porous thermal conductivity ratio and
\end{abstract}


permeability of porous layer on conjugate natural convection heat transfer in an open-ended porous-partially-filled square cavity, for the first time. It is found that these factors all influence the patterns of flow field and temperature field significantly. Especially, there exist some critical values. A small offset from them will cause a substantial change of heat and mass transfer. Sometimes the change trends are completely reversed. The present results may provide useful theoretical guides for the relevant practical applications.

Key words: Conjugate heat transfer; porous media; natural convection; open-ended cavity; lattice Boltzmann method

\section{Introduction}

Natural convection in an open-ended cavity is an important research prototype in thermal science and engineering as it can be used to deepen our insights into many practical applications, such as solar thermal receivers, building ventilation and energy saving [1-3]. Until now there have been numerous publications on this topic. On experimental exploration, recently Montiel-Gonzalez et al. [4] revealed the effects of natural convection and thermal radiation on a solar open cubic cavity-type receiver. The authors observed that variable thermophysical properties of working fluid would influence the results significantly. Natural convection in a horizontal open-ended axisymmetric cavity was investigated experimentally by the holographic interferometry technique [5]. The investigators mapped the isotherms as a function of the temperature on the 
hot wall and the cavity spacing. Heat transfer in an open-ended vertical eccentric enclosure was reported in [6]. Through analyzing their experimental data, the authors found that the heat transfer coefficient did not change monotonically with the eccentric ratio. Meanwhile, numerical efforts were also carried out. Natural convection of $\mathrm{Al}_{2} \mathrm{O}_{3}$-water nanofluid in an open-ended enclosure was numerically investigated in [7]. The purpose of [7] was to reveal the influence of magnetic force on heat transfer of nanofluid in an open-ended cavity. Marangoni convection in an open-ended cavity was numerically simulated by Saleem et al.[8]. The authors discussed the effect of thermocapillary forces on natural convection. They also carried out entropy generation analysis. Mohamad et al. [11] studied the influence of aspect ratio on natural convection in open-ended cavities. The numerical results showed the rate of heat transfer deceases asymptotically against increasing of aspect ratio. In [9], the authors considered the scenario where an open-ended cavity was completely filled by porous media. The effects of porosity and permeability of porous media on natural convection were investigated numerically. Boetcher and Sparrow [10] compared some available numerical techniques and stressed the importance of appropriate boundary conditions. Only a few latest studies on this topic are cited here as the total number of the relevant literature is very huge.

In a lot of practical applications, an enclosure is usually partially filled with porous media, e.g. a room with multi-layer building materials[12] or a new type of solar energy receiver [13] . In such scenarios, conjugate heat transfer 
must be considered appropriately on the interface between porous media and working fluid, which significantly increases the complicity of an investigated domain $[14,15]$. So far there have been a number of literature on conjugate natural convection heat transfer in an enclousre partially filled with porous media. Beckermann et al. $[16,17]$ perhaps are the pioneers in this field. They investigated conjugate natural convection heat transfer in a vertical cavity, in which a porous layer was placed. Their experimental and numerical data showed for a high effective-thermal-conductivity porous layer, natural convection in the porous layer was suppressed, while the isotherms bent sharply on the fluid/porous interface. Natural convection in a square cavity in which differentially heated vertical walls were covered with thin porous layers was studied in [18]. It was observed that the main effect of the porous layer was to decrease convective heat transfer. Singh et al. [19] considered a three dimensional domain. Their numerical results indicated that penetration of the fluid into the porous region depended strongly on the Darcy number $(D a)$ and Rayleigh number $(R a)$. The influence of a horizontal partial porous partition on natural convection in a square cavity was numerically studied in [20]. The authors claimed that the porous partition would have obvious influence on heat transfer if its size was thick enough. Song and his cooperators [21] experimentally studied natural convection within a rectangular enclosure partially filled with an anisotropic porous medium. They also carried out numerical simulation to reveal the effect of anisotropic permeability on heat transfer characteristics. Natural convective heat transfer from a cylinder in an 
enclosure partly filled with a porous medium was reported in [22]. Their work showed how the mean heat transfer rate from the cylinder was affected by the size of the fluid gap at the top of the enclosure. Transient natural convection in a cavity partially filled with porous media was investigated, too [23]. In [23], four different configurations were considered to show their influences on the transient processes. As conjugate heat transfer between working fluid and porous media is a challenge for numerical heat transfer research, Arpino et al. [24] tried to developed a more efficient numerical approach to solve the difficulty. Recently, Sheremet and Trifonova[25] numerically investigated unsteady conjugate natural convection in a vertical enclosure partially filled with porous media. The effect of the Darcy number $(D a)$ was discussed in their research. A latest comprehensive review on this topic was presented in [14]. For traditional numerical techniques, to appropriately treat conjugate heat transfer across fluid/porous interface is a great challenge, especially for complicate geometry [14]. Unfortunately, for numerous practical energy systems, conjugate heat transfer plays an important role $[26,27]$.

Through the above literature survey, it is clear that until now there is no open publication on conjugate natural convection heat transfer in an open-ended enclosure partially filled with porous media, although it is important in energy engineering and civil engineering. The purpose of the present work is to bridge such gap. In the present work, the lattice Boltzmann (LB) method [28] is adopted as a numerical tool to avoid complicated treatments for conju- 
gated fluid/porous interfaces in traditional numerical approaches. Until now there have emerged some research on modelling conjugate heat transfer by the LB method [29-36]. They share a common advantage that interfaces can be treated easily. Karani and Huber [29] proposed a LB model for conjugate heat transfer between fluid flow and solid walls. In their model, any interface between fluid and solid phases was not necessarily treated explicitly. Hu et al. designed a LB model for conjugate heat transfer between fluid flow and solid walls, based on the so-called immersed boundary method to avoid specific treatment on interfaces [30]. An extrapolation LB scheme was developed in [31]. The conjugate heat transfer across curved interfaces can be treated more accurately by their scheme. In [32], the concept "enthalpy" in thermodynamics was introduced to simplify the treatment of conjugate heat transfer on interfaces between fluid and solid. Pareschi and his cooperators [33] extended the so-called Grad's boundary treatment to deal with conjugate heat transfer. Lu et al. [34] found out a different way to deal with conjugate heat transfer between fluid and solid phases. In their LB model, a correction step was introduced to reflect the effect of interfaces on heat flux. The LB models for conjugate heat transfer between porous media and solid walls were presented in $[35,36]$. A brief review on the advantages and disadvantages of the available LB models for conjugate heat transfer can be found in [32,36]. Obviously, the available LB models all do not cover the topic of conjugate fluid/porous interface with arbitrary heat capacitance ratio (heat capacitance $C$ is the product of density and specific heat capacity). Therefore, the purpose of the present 
study is twofold: Firstly, a new LB model is proposed to remedy the shortcoming. Secondly, with the aid of this new LB model, the effects of thickness of porous layer, fluid-to-porous thermal conductivity ratio and permeability of porous layer on the characteristics of natural convection heat transfer in an open-ended porous-partially-filled square cavity are discussed for the first time.

\section{Governing equations}

The governing equations for heat transfer in an open-ended enclosure, where porous media and pure fluid coexist, can be written as $[14,25]$

$$
\begin{gathered}
\partial_{\alpha} u_{\alpha}=0 \\
\partial_{t} u_{\alpha}+u_{\beta} \partial_{\beta} \frac{u_{\alpha}}{\varepsilon}=-\partial_{\alpha} \varepsilon p+\partial_{\beta} \nu_{e}\left(\partial_{\alpha} u_{\beta}+\partial_{\beta} u_{\alpha}\right)+F_{\alpha} \\
\sigma \partial_{t} T+u_{\alpha} \partial_{\alpha} T=\partial_{\alpha} \kappa_{e} \partial_{\alpha} T .
\end{gathered}
$$

where $u_{\alpha}\left(u_{\beta}\right), p, T$ and $\varepsilon$ are fluid velocity, pressure, temperature and porosity of porous media, respectively. For a pure fluid zone, $\varepsilon=1$. In addition, $\nu_{e}$ and $\kappa_{e}$ are the effective kinematic viscosity and thermal diffusivity. Their 
definitions can be found in [37]. There are the following relationships for them

$$
\begin{aligned}
& \nu_{e}=\left\{\begin{array}{l}
\nu_{f}, \quad \text { in fluid zone } \\
\nu_{\text {porous }}, \text { in porous media zone }
\end{array}\right. \\
& \kappa_{e}=\left\{\begin{array}{l}
\kappa_{f}, \quad \text { in fluid zone } \\
\kappa_{\text {porous }}, \text { in porous media zone }
\end{array}\right.
\end{aligned}
$$

where the subscripts $f$ and porous indicate pure working fluid and fluidsaturated porous media, respectively. Usually, it is assumed that $\nu_{\text {porous }}=\nu_{f}$ [37]. The parameter $\sigma$ has the following choices

$$
\sigma=\left\{\begin{array}{l}
1, \text { in fluid zone } \\
\sigma_{\text {porous }}, \text { in porous media zone }
\end{array}\right.
$$

$\sigma_{\text {porous }}$ is determined by the heat capacitance ratio of porous matrix to saturating fluid $[14,25]$. 
For convectional flow, the force $F_{\alpha}$ represents

$$
F_{\alpha}=\left\{\begin{array}{l}
-\varepsilon g_{\alpha} \beta \Delta T, \quad \text { in fluid zone } \\
-\frac{\varepsilon \nu_{e}}{K} u_{\alpha}-\frac{1.75}{\sqrt{150 \varepsilon K}}\left|u_{\alpha}\right| u_{\alpha}-\varepsilon g_{\alpha} \beta \Delta T, \quad \text { in porous media zone }
\end{array}\right.
$$

where $g_{\alpha}, \beta, \Delta T$ and $K$ are the gravity, thermal expansion coefficient, temperature difference and permeability of porous media, respectively. Compared with a pure working fluid zone, two additional drag force terms exist in a porous layer according to the so-called Forchheimer-extended Darcy equation [14]. What should be stressed is that Eq. (3) is valid only when working fluid and fluid-saturated porous media can always keep local thermal equilibrium status.

In addition, on a fluid/porous interface, the following conjugate restriction should be satisfied [14,23-25]:

$$
\begin{gathered}
T_{f}=T_{\text {porous }} \\
n_{\alpha}\left[\nabla_{\alpha} T\right]_{f}=n_{\alpha}\left[R_{k} \nabla_{\alpha} T\right]_{\text {porous }}
\end{gathered}
$$

where $n_{\alpha}$ is normal to the interface, and [ $]_{\text {porous }}$ and []$_{f}$ indicate the parameters at the porous/fluid side of the interface. The parameter $R_{k}$ indicates the porous-to-fluid thermal conductivity ratio. For the popularly used numerical methods, specific treatments are required to ensure Eqs. (8)-(9) to be 
held exactly on fluid/porous interfaces [14,23-25]. Especially, for complicated interfaces, it will be a great challenge to identify the normal direction $n_{\alpha}$ [24].

\section{LB model for conjugate heat transfer across fluid/porous inter- faces}

As shown in our previous work [36,38], if we can establish an appropriate LB distribution function for the temperature field, the LB method can avoid the complicated treatments on conjugate fluid/porous interfaces (Eqs.(8)-(9)) which is a great challenge for traditional numerical approaches. In the LB community, a thermal model proposed by Guo et al. [37] is popularly used for simulating heat transfer in porous media. However, as in Guo's model the parameter $\sigma$ in Eq.(3) should be homogeneous over the whole investigated domain, it can not be used to simulate conjugate heat transfer across a fluid/porous interface with arbitrary heat capacitance ratio (namely $\sigma_{\text {porous }} \neq 1$ in Eq.(6))[39]. In this section, for simplicity we take a single-relaxation-time (SRT) LB model as an example to show how to overcome the difficulty. Its multi-relaxation-time (MRT) counterpart can be established in the same way.

For conjugate heat transfer, only the LB model for temperature field that proposed in [37] should be re-established, consequently for clarity in the present work we only explain how to construct a new LB model for temperature field governed by Eq. (3). The flow field governed by Eqs. (1)-(2) can be solved by 
the same way used in [37].

\subsection{Evolving equation}

The LB evolving equation for temperature field reads:

$$
g_{j}\left(x_{\alpha}+c e_{j \alpha} \Delta t, t+\Delta t\right)-g_{j}\left(x_{\alpha}, t\right)=-\tau_{T}^{-1}\left[g_{j}\left(x_{\alpha}, t\right)-g_{j}^{(e q)}\left(x_{\alpha}, t\right)\right] .
$$

In Eq.(10) $\tau_{T}$ is the dimensionless relaxation time for the pseudo-particle distribution $g_{j}\left(x_{\alpha}, t\right)$ at space $x_{\alpha}$ and instant $t . c e_{j \alpha}$ denotes the discrete velocities and $\Delta t$ means the time interval in the LB evolving equation.

In order to model conjugate heat transfer across a fluid/porous interface with arbitrary heat capacitance ratio, a new type of equilibrium distribution $g_{j}^{(e q)}$ in Eq.(10) is proposed:

$$
g_{j}^{(e q)}=\left\{\begin{array}{l}
T\left(\sigma-\sigma_{0}\right)+\omega_{j} T\left(\sigma_{0}+\frac{c e_{j \alpha} u_{\alpha}}{c_{s}^{2}}\right), \quad j=0 \\
\omega_{j} T\left(\sigma_{0}+\frac{c e_{j \alpha} u_{\alpha}}{c_{s}^{2}}\right), \quad j \neq 0
\end{array}\right.
$$

where $\omega_{j}$ represents the weight coefficients. $\sigma_{0}$ is a reference value of $\sigma$ so $\sigma_{0}$ is a constant within the whole investigated domain. The parameter $c_{s}$ satisfies $c_{s}^{2} \delta_{\alpha \beta}=\sum_{j} \omega_{j} c^{2} e_{j \alpha} e_{j \beta}[40]$. 
The temperature $T$ is obtained by

$$
T=\frac{\sum_{j} g_{j}}{\sigma} .
$$

and the effective thermal diffusivity $\kappa_{e}$ is given by

$$
\kappa_{e}=\sigma_{0}\left(\tau_{T}-1 / 2\right) c_{s}^{2} \Delta t
$$

According to Eq.(13), it is clear that in the present model the effective thermal diffusivity depends on $\sigma_{0}$, rather than $\sigma$ in Guo's model (c.f. Eq.(30) in [37]). As shown below, it is the key to model conjugate heat transfer across fluid/porous interface with arbitrary heat capacitance ratio (i.e. $\sigma_{\text {porous }} \neq 1$ in Eq.(6)).

\subsection{Multiscale expansion and recovered macroscopic equation}

Equation (10) can be expanded in Taylor series as [28]

$$
\Delta t\left(\partial_{t}+c e_{j \alpha} \nabla_{\alpha}\right) g_{j}+\frac{\Delta t^{2}}{2}\left(\partial_{t}+c e_{j \alpha} \nabla_{\alpha}\right)^{2} g_{j}+\frac{1}{\tau_{T}}\left[g_{j}-g_{j}^{(e q)}\right]=O\left(\Delta t^{3}\right)
$$

Introducing the multiscale expansion $\partial_{t}=\epsilon \partial_{t 1}+\epsilon^{2} \partial_{t 2}, \nabla_{\alpha}=\epsilon \nabla_{\alpha 1}$ and $g_{j}=$

$g_{j}^{(e q)}+\epsilon g_{j}^{(1)}+\epsilon^{2} g_{j}^{(2)}+O\left(\epsilon^{3}\right)[37]$, we can sort Eq. (14) in terms of $\epsilon$ and $\epsilon^{2}$ as

$$
\left(\partial_{t 1}+c e_{j \alpha} \nabla_{\alpha 1}\right) g_{j}^{(e q)}=-\frac{g_{j}^{(1)}}{\Delta t \tau_{T}}+O(\epsilon)
$$




$$
\partial_{t 2} g_{j}^{(e q)}+\left(\partial_{t 1}+c e_{j \alpha} \nabla_{\alpha 1}\right)\left[\left(1-\frac{1}{2 \tau_{T}}\right) g_{j}^{(1)}\right]=-\frac{g_{j}^{(2)}}{\Delta t \tau_{T}}+O\left(\epsilon^{2}\right) .
$$

With the symmetry properties of the lattice $\sum_{j} \omega_{j} c e_{j \alpha}=0$ and $\sum_{j} \omega_{j} c e_{j \alpha} c e_{j \beta}=$ $c_{s}^{2} \delta_{\alpha \beta}$ we can obtain

$$
\begin{gathered}
\sum_{j} g_{j}^{(e q)}=\sigma T, \\
\sum_{j} c e_{j \alpha} g_{j}^{(e q)}=T u_{\alpha}, \\
\sum_{j} c e_{j \alpha} c e_{j \beta} g_{j}^{(e q)}=\sigma_{0} T c_{s}^{2} \delta_{\alpha \beta} .
\end{gathered}
$$

Please bear in mind that the second moment of $g^{(e q)}$ (namely Eq.(19)) is different from that of Guo' model[37].

With the aid of Eqs.(17)-(19), as well as $\sum_{j} g_{j}^{(1)}=\sum_{j} g_{j}^{(2)}=0$, the summation of Eqs.(15)-(16) over the discrete direction $e_{j \alpha}$ reads

$$
\begin{gathered}
\partial_{t 1} \sigma T+\nabla_{\alpha 1} T u_{\alpha}=0+O(\epsilon), \\
\partial_{t 2} \sigma T+\nabla_{\alpha 1}\left[c_{s}^{2}\left(\frac{1}{2}-\tau_{T}\right) \Delta t \nabla_{\alpha 1} \sigma_{0} T\right]=0+O\left(\epsilon^{2}\right) .
\end{gathered}
$$

Because $\sigma_{0}$ is a constant across the whole investigated domain, $\nabla_{\alpha 1} \sigma_{0} T=$ $\sigma_{0} \nabla_{\alpha 1} T$. Accordingly Eq.(21) can be re-written as

$$
\partial_{t 2} \sigma T+\nabla_{\alpha 1}\left[\sigma_{0} c_{s}^{2}\left(\frac{1}{2}-\tau_{T}\right) \Delta t \nabla_{\alpha 1} T\right]=0+O\left(\epsilon^{2}\right)
$$

Combining Eqs.(20) and (22), we can obtain the final recovered macroscopic 
governing equation for temperature field

$$
\partial_{t} \sigma T+\nabla_{\alpha} T u_{\alpha}=\nabla_{\alpha} \kappa_{e} \nabla_{\alpha} T+O\left(\epsilon^{2}\right)
$$

where $\kappa_{e}=\sigma_{0} c_{s}^{2}\left(\tau_{T}-\frac{1}{2}\right) \Delta t$. It is obvious that Eq.(23) can match Eq.(3) exactly.

However, in Guo's model [37], $\kappa_{e}=\sigma c_{s}^{2}\left(\tau_{T}-\frac{1}{2}\right) \Delta t$, which implies the local effective thermal diffusivity depends on the porous-to-fluid heat capacitance ratio. It is non-physical and this non-physical shortcoming places an artificial restrict that $\sigma$ should be homogenous over the whole domain, as pointed by Guo et al. [37]. Consequently, for conjugate fluid/porous interface with arbitrary heat capacitance ratio, namely $\sigma_{\text {porous }} \neq 1$, Guo's model becomes invalid. As shown in our numerical tests below, if $\sigma_{\text {porous }} \neq 1$, Guo's model will diverge.

As demonstrated in our previous work[38], for a LB evolving equation like Eqs.(10)-(11), at any cross section within the investigated domain, the following equalities can be guaranteed automatically

$$
\begin{gathered}
T_{+}=T_{-} \\
n_{\alpha}\left[\kappa_{e} \nabla_{\alpha} T\right]_{+}=n_{\alpha}\left[\kappa_{e} \nabla_{\alpha} T\right]_{-}
\end{gathered}
$$

where [ ]+ and [ ] indicate the parameters at each side of the cross section. In addition, for fluid-saturated porous media, there is $R_{k}=\frac{\rho_{f} \kappa_{\text {porous }}}{\rho_{f} \kappa_{f}}=\frac{\kappa_{\text {porous }}}{\kappa_{f}}$ 
[37]. Consequently, along a fluid/porous interface, being divided by $\kappa_{f}$, Eq.(25) can be re-written as

$$
n_{\alpha}\left[\nabla_{\alpha} T\right]_{f}=n_{\alpha}\left[\frac{\kappa_{\text {porous }}}{\kappa_{f}} \nabla_{\alpha} T\right]_{\text {porous }}=n_{\alpha}\left[R_{k} \nabla_{\alpha} T\right]_{\text {porous }}
$$

Please notice in Eq.(26) the subscripts + and - are replaced by $f$ and porous for clarity. Namely, the conjugate restriction can be guaranteed automatically by the present LB model. We do not need any explicit treatment to satisfy Eqs.(8)-(9).

\section{Numerical validation}

In order to validate the present numerical approach, three simple but nontrivial benchmark tests are adopted. The first one is natural convection in an open-ended square cavity investigated in [11], which can validate the accuracy of the present numerical approach for open boundaries as an appropriate boundary treatment is very important for the LB method. The second one is conjugate mixed convection heat transfer in a partially porous cavity investigated in [14], which can check the applicability of the present numerical approach for conjugate heat transfer modelling at fluid/porous interfaces. The last one is transient conjugate heat conduction in three-layered stratified media [32], which can show the reliability of the present numerical approach for simulating conjugate heat transfer of a thermodynamically heterogenous sys- 
Table 1

Average Nusselt number on the left wall with various $R a$.

\begin{tabular}{ccc}
\hline$R a$ & {$[11]$} & present \\
\hline$R a=10^{4}$ & 3.37 & 3.2509 \\
$R a=10^{5}$ & 7.33 & 7.2251 \\
$R a=10^{6}$ & 14.38 & 14.4142 \\
\hline
\end{tabular}

tem. The grid resolution is $100 \times 100$ for all simulations in the present work. What should be mentioned is that all simulations in the present work are based on dimensionless quantities. The non-dimensional process for the LB method can be found in [28].

\subsection{Natural convection in an open-ended square cavity}

Natural convection in an open-ended square cavity, which was numerically investigated in [11], is adopted to validate the accuracy of the present numerical approach for open-ended boundary treatment. As in [11] there is no porous filled in the cavity, we set the Darcy number $D a=10^{6}$, the porosity $\varepsilon=0.999$ and $\sigma=1.0$ for the whole domain. The LB boundary scheme used in [11] is adopted here, too.

Figures 1-2 depict the streamlines and isotherms at various $R a$. They are identical to those presented in [11] (c.f. Figs. 2-10 in [11]). For quantitative comparison, Table 1 lists the average Nusselt number $N u$ at the vertical wall. The present results agree well with the data in [11]. 


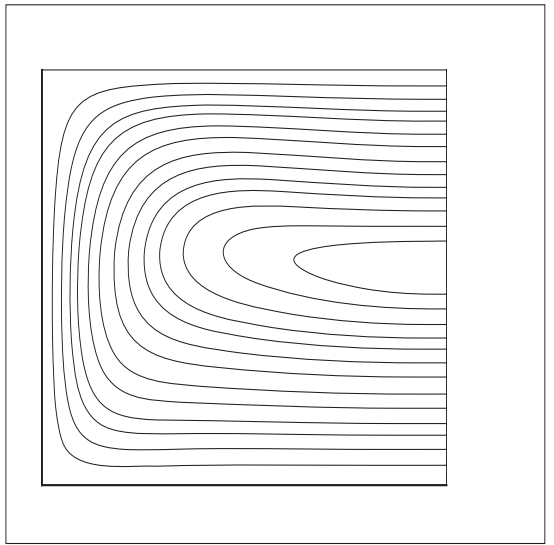

(a)

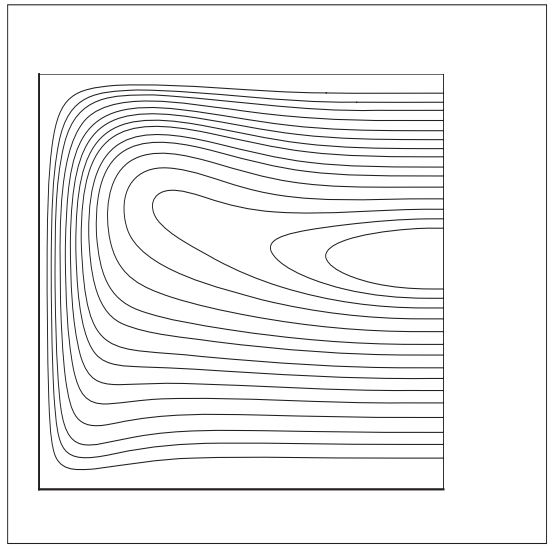

(b)

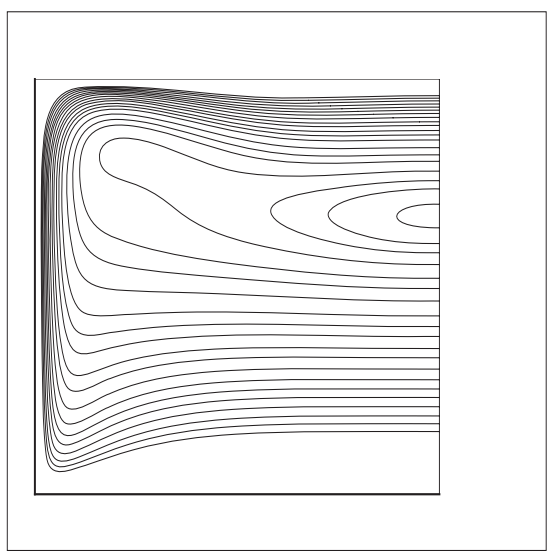

(c)

Fig. 1. Streamlines at (a) $R a=10^{4}$ (b) $R a=10^{5}$ and (c) $R a=10^{6}$.

4.2 Conjugate mixed convection heat transfer in a partially porous square cavity

Conjugate mixed convection heat transfer in a partially porous square cavity investigated in [14] is adopted as the second benchmark test, which can validate the capability of the present numerical approach for conjugate heat transfer modelling across a fluid/porous interface. The parameters are $D a=10^{-3}$ and $\varepsilon=0.6$. The porous-to-fluid heat capacitance ratio is unity, namely $\sigma_{\text {porous }}=1.0$, the same as that in $[14]$. 


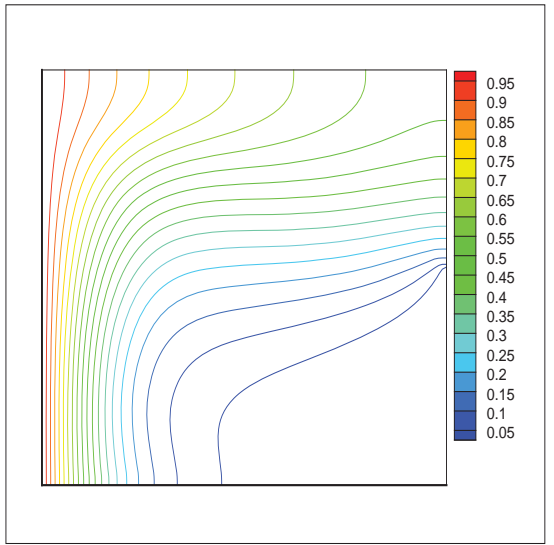

(a)

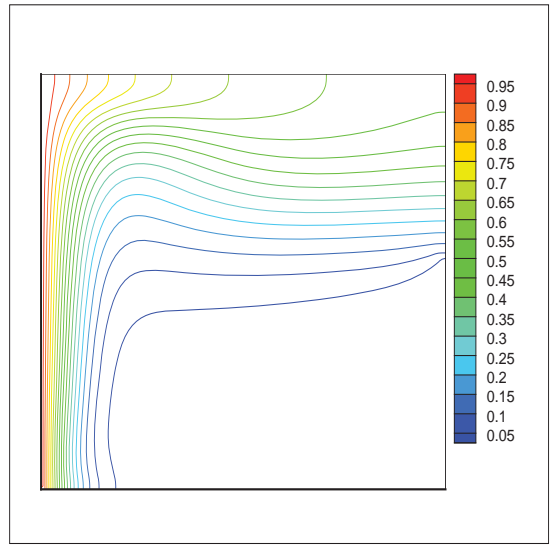

(b)

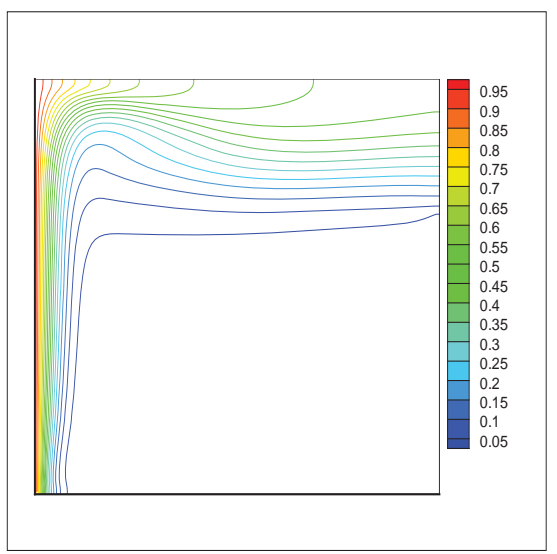

(c)

Fig. 2. Isotherms at (a) $R a=10^{4}$ (b) $R a=10^{5}$ and (c) $R a=10^{6}$.

Table 2

Average Nusselt number on the hot wall with various $R_{k}$.

\begin{tabular}{ccc}
\hline$R k$ & {$[14]$} & present \\
\hline 0.1 & 3.73 & 3.6168 \\
1 & 1.23 & 1.1859 \\
10 & 0.19 & 0.1867 \\
\hline
\end{tabular}

Figure 3 illustrates the profiles of mid-height velocity across the cavity and temperature along the fluid/porous interface at various $R_{k}$. Table 2 lists the average Nusselt number $N u$ at the hot wall of the enclosure. One can observe that the present numerical results agree well with those in [14]. 


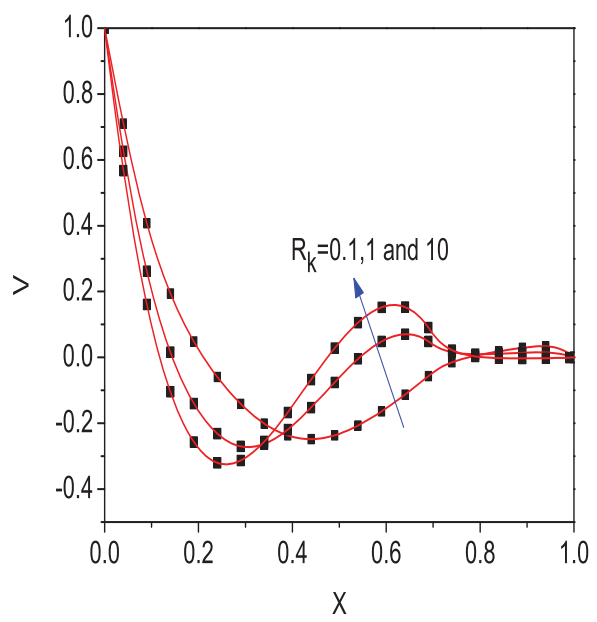

(a)

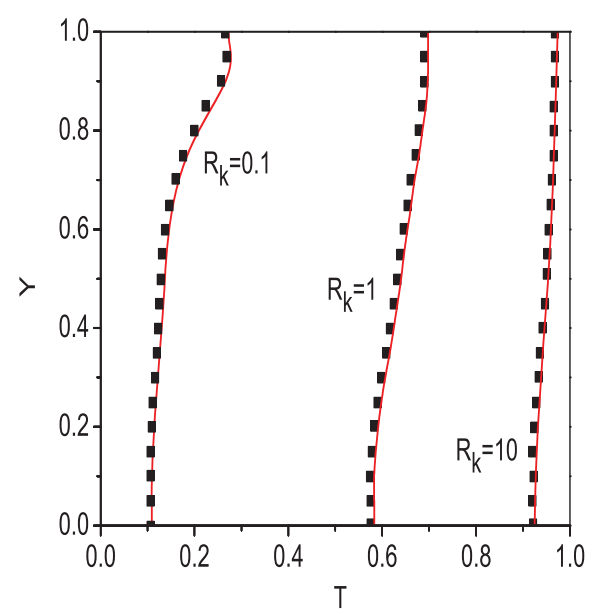

(b)

Fig. 3. Profiles of (a) mid-height velocity across cavity and (b) temperature on fluid/porous interface at various $R_{k}$ : solid line-present results; squares-data in [14].

\subsection{Transient conjugate heat conduction in three-layered stratified media}

The schematic configuration of transient conjugate heat conduction in threelayered stratified media is depicted by Fig. 4. The fluid keeps stationary as no convection is considered. At time $t=0$, the temperature over the whole domain is identical, as $T_{c}=0$. Since $t>0$, the temperature on the downside 


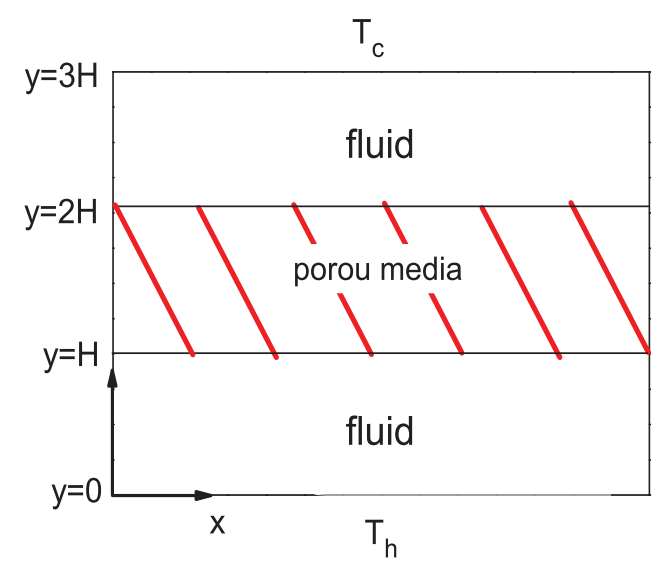

Fig. 4. Schematic configuration of three-layered stratified media.

is elevated and fixed at $T_{h}=1.0$. The vertical sides of the investigated domain both are adiabatic. In our simulation $D a=10^{-6}$ and $\varepsilon=0.1$. In addition, $R_{k}=0.1$ and $\sigma_{\text {porous }}=2.0$.

Figure 5 illustrates the temperature profiles along the vertical central line at different instants until the steady status obtained by the present approach, compared with the numerical data given in [32]. The present results agree well with the results in Ref.[32], which further demonstrates the present model's reliability for simulating conjugate heat transfer in thermodynamically heterogeneous systems.

\section{Results and discussion}

The configuration of the open-ended square cavity is illustrated by Fig. 6 . The dimensionless side length of the square cavity is $L=1.0$. A porous layer with thickness $d$ is placed against the hot vertical solid wall. The dimensionless 


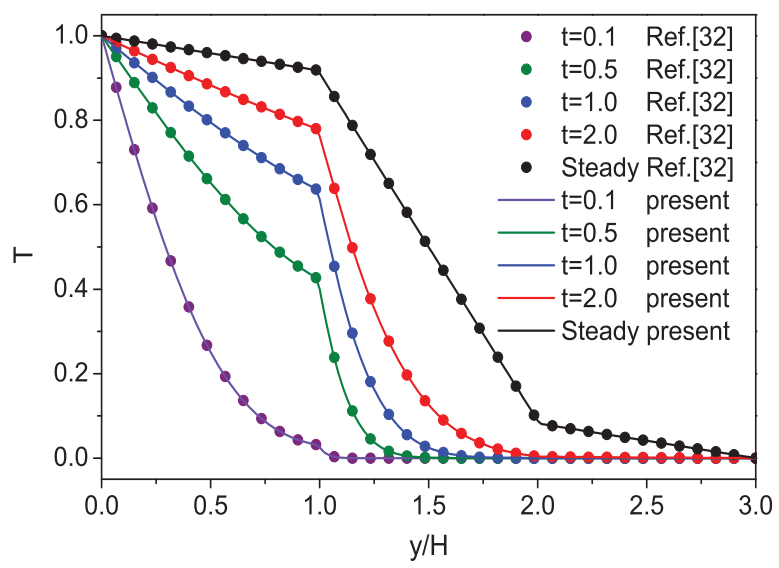

Fig. 5. Temperature profiles along the vertical central line at various dimensionless time: solid lines-present results, dots-solutions in Ref.[32].

temperature of the hot wall is $T_{h}=1.0$. The Prandlt number of the working fluid $\operatorname{Pr}=0.7$. The upper and lower solid walls are adiabatic. The cavity exchange heat and mass with the environment through the left open side. The ambient temperature is $T_{0}=0$. According to [11], at the left open boundary, there are the following relationships

$$
\begin{aligned}
& T=T_{0}, \quad u>0 \\
& \frac{\partial T}{\partial x}=0, \quad u<0
\end{aligned}
$$

The LB boundary treatment proposed in [9] is used in the present simulation.

\subsection{Effect of thickness of porous layer}

In order to reveal the effect of thickness of porous layer $d$, here we introduce a dimensionless quantity $d / L$, namely the ratio of the thickness of porous layer 


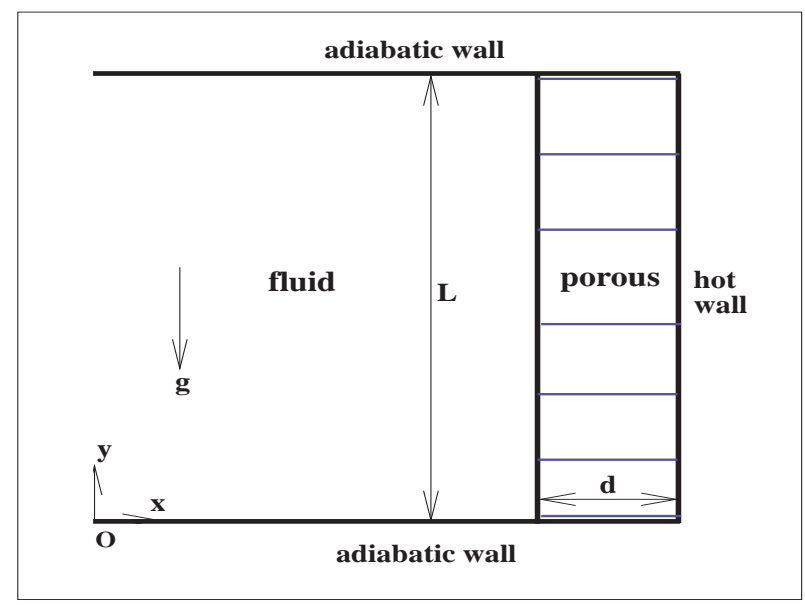

Fig. 6. Schematic configuration of open-ended enclosure partially filled with porous media.

to the length of the cavity. Such dimensionless quantity is more useful for comparable analysis between different applications. In this subsection we set $D a=10^{-3}, \varepsilon=0.6, R a=10^{5}, \sigma_{\text {porous }}=1$ and $R_{k}=1$, while $d / L$ varies between 0.1 and 0.5 .

Figure 7 depicts the streamlines at various $d / L$. The dashed line in the figure indicates the position of the porous/fluid interface. One can observe that the flow enters from the lower half portion of the cavity and leaves from the upper half of the opening for all scenarios. Different from its counterpart in a closed square cavity where there is only one big vortex (c.f. Fig.2), due to the existence of the porous layer, there are more than one vorticity in the openended domain: a big counterclockwise vorticity near the open boundary and a large clockwise one in the vicinity of the porous/fluid interface. The latter is caused by the relative high drag resistance of the porous layer. Consequently, with the thickness of the porous layer decreasing, the center of the clockwise vortex moves towards the hot wall and its size is compressed obviously. For a 
very thin porous layer (e.g. $d / L=0.1$ ), it will split into two small vortices. On the contrary, the counterclockwise vortex expands against $d$ diminishing. When the counterclockwise vortex approaches to the hot wall, the streamlines will tilt upwards due to the strong thermal buoyant force in the neighbour of the hot wall. The thickness of the porous layer can influence the flow pattern significantly.

Figure 8 shows the profile of horizontal velocity along the fluid-porous interface and of mid-height velocity across the cavity, which can indicate more detailed information on the flow pattern in the cavity. It is not surprised that a thicker porous layer can make the distribution of velocity more non-uniform in the domain. Moreover, according to this figure, one can observe that a thicker porous layer will suppress the circulation in the fluid zone while enhance the circulation within the porous area as establishment and development of free convectional flow require sufficient space.

The isotherms at various $d / L$ are illustrated by Fig. 9. Generally, the dominant heat transfer mechanism in the porous layer is heat conduction as the isotherms in the porous zone are nearly parallel with the vertical hot wall, especially for a very thin porous layer (e.g. $d / L=0.1$ ). With $d / L$ increasing, the isotherms in the porous zone become to be disturbed, which implying the share of convective heat transfer become increasing. On the contrary, in the fluid zone, more isotherms become horizontally, which indicates convective heat transfer is predominant, when the porous layer becomes thinner. The 
variation of isotherms is consistent with that of streamlines plotted by Fig. 7. In addition, more area in the cavity will become cold with $d / L$ decreasing. It is caused by the counterclockwise vortex near the opening, whose strength is enhanced by a thin porous layer, so more cold fluid is sucked from the environment.

Figure 10 illustrates the variation of the average Nusselt number at various $d / L$. One can find that the average Nusselt number is a monotonically decreasing function against $d / L$. Within the range $d / L=0.2-0.5, N u$ diminishes almost linearly. However, when $d / L=0.1-0.2$, a sharp fall appears, which implies there is a significant change of heat transfer pattern. The profile of local Nusselt number along the hot wall depicted by Fig. 11 can show it more clearly. According to Fig. 11, one can observe that for a thick porous layer, the most intensive heat transfer emerges at the upper half portion of the hot wall. However, for a very thin porous layer (e.g. $d / L=0.1$ ), the most intensive heat transfer appears at the lower half part of the vertical wall. The substantial change of heat transfer pattern is consistent with the variation of flow pattern. As shown by Fig.7 (c), for a very thin porous layer like $d / L=0.1$, the vortex in the porous zone breaks, which completely alter heat transfer pattern. Through the present study, it can be concluded that there is a critical thickness of the porous layer for natural convection in the open-ended cavity, across which the flow and heat transfer patterns will change significantly. Therefore, for practical applications, it is crucial to find such critical thickness. 


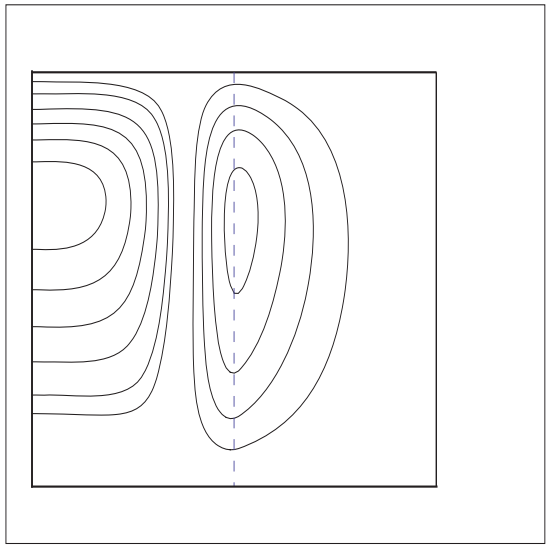

(a)

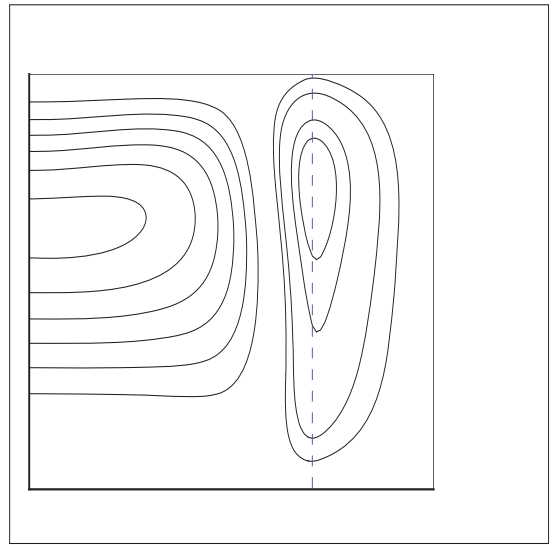

(b)

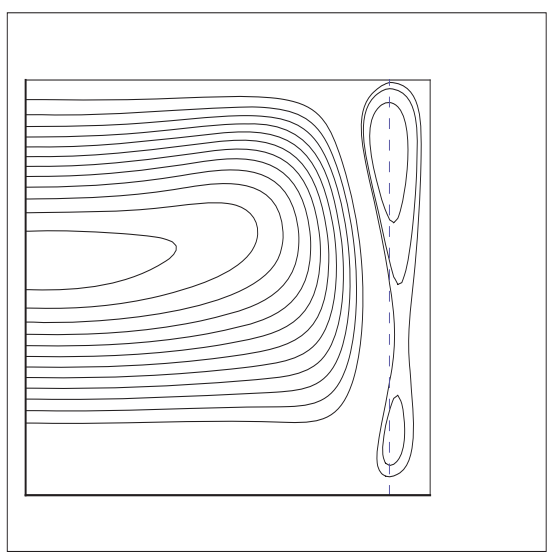

(c)

Fig. 7. Streamlines at (a) $d / L=0.5$ (b) $d / L=0.3$ and (c) $d / L=0.1$.

In [18], it was observed that in a closed cavity partially filled by a thin porous layer, the thin porous layer would decrease convective heat transfer. However, through the present work, it is found such conclusion can not be extended to its open-ended counterpart. As shown by Fig. 10, in the present open-ended cavity, a thin porous layer can enhance convective heat transfer. 


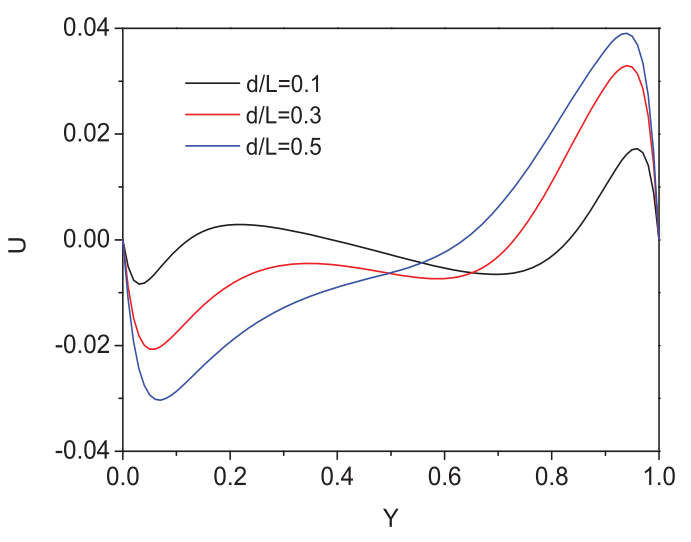

(a)

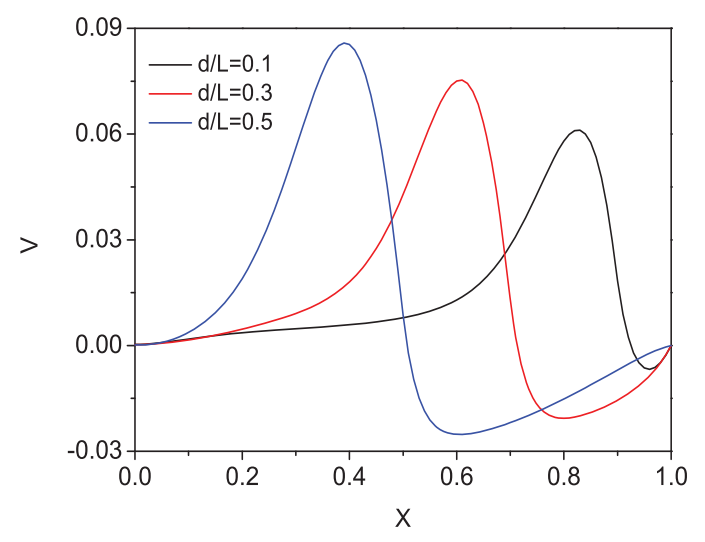

(b)

Fig. 8. Profile of (a) horizontal velocity along the fluid-porous interface and (b) mid-height velocity across cavity.

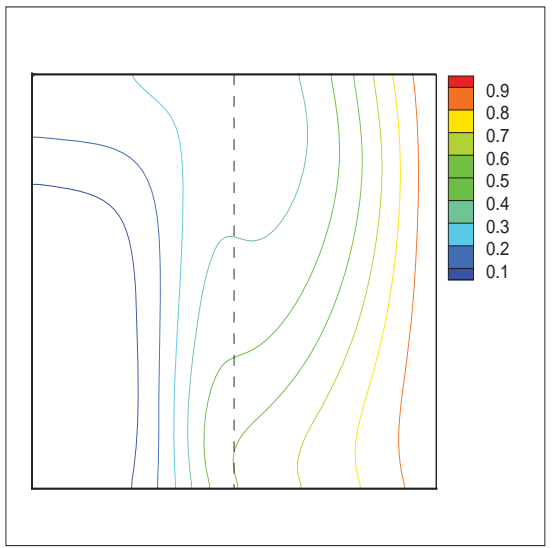

(a)

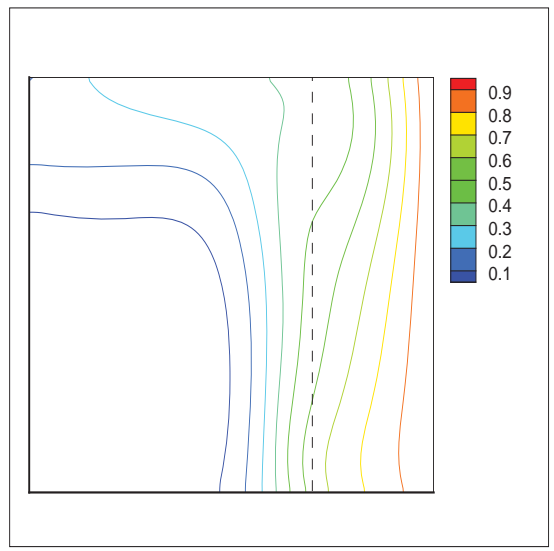

(b)

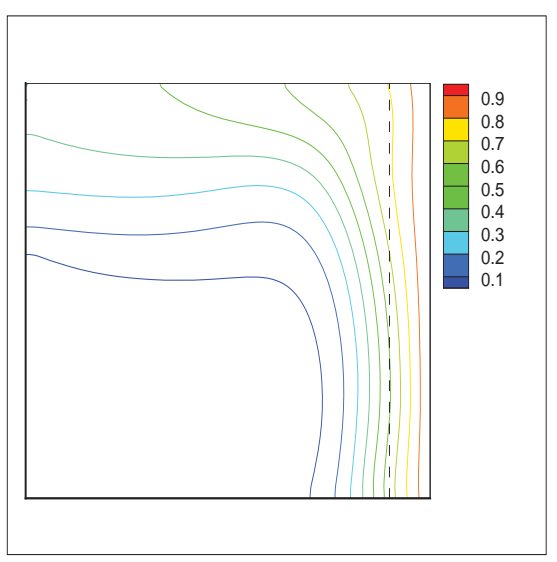

(c)

Fig. 9. Isotherms at (a) $d / L=0.5$ (b) $d / L=0.3$ and (c) $d / L=0.1$. 


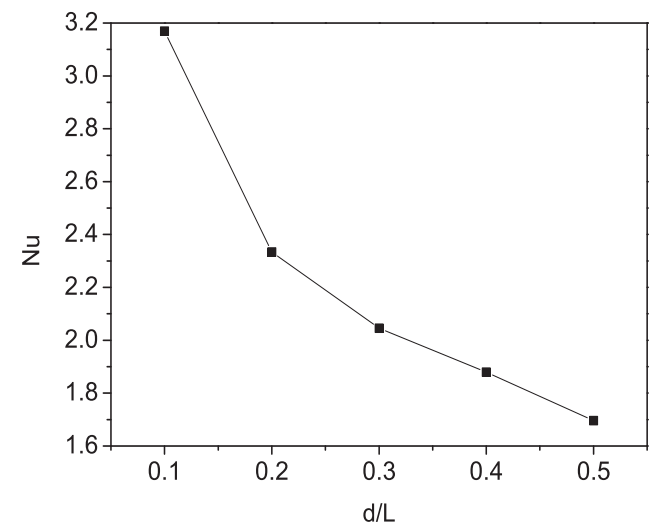

Fig. 10. Average Nusselt number versus thickness of porous layer.

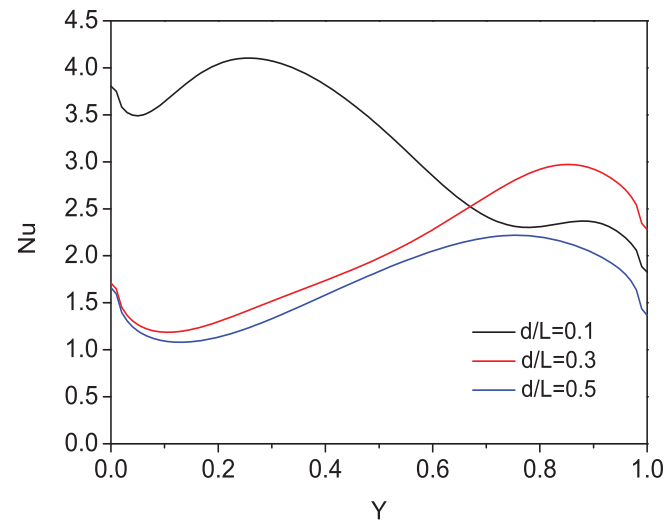

Fig. 11. Local Nusselt number along the hot wall at various $d / L$.

\subsection{Effect of porous-to-fluid thermal conductivity ratio}

In order to reveal the effect of porous-to-fluid thermal conductivity ratio $R_{k}$, in this subsection we set $D a=10^{-3}, \varepsilon=0.6, R a=10^{5}, \sigma_{\text {porous }}=1.0$ and $d / L=0.3$, while $R_{k}$ varies between 0.1 and 10 .

Figure 12 plots the streamline at different $R_{k}$. It is clear that the flow pattern is altered considerably by $R_{k}$. When $R_{k}$ is very small (e.g. $R_{k}=0.1$ ), the cold fluid from the environment can successfully penetrate the interface and form 
a great clockwise vortex near the geometric center of the cavity. However, for a high $R_{k}$ (e.g. $R_{k}=10$ ), the cold fluid fails to do so and there emerge two vortices: a counterclockwise vortex near the open boundary and a clockwise one in the vicinity of the porous/fluid interface. In the fluid zone, the flow direction at a low $R_{k}$ is completely reversed, as compared with its high $R_{k}$ counterpart.

The corresponding isotherms are presented by Fig. 13. Please bear in mind that an increment of $R_{k}$ indicates increasing of effective thermal conductivity of the porous layer. Consequently, for a high $R_{k}$ (e.g. $\left.R_{k}=10\right)$, the thermal resistance of the porous layer is much smaller than the fluid zone. Accordingly, the temperature gradient in the porous zone is slight and the distribution of temperature looks very uniform within the porous layer. Meanwhile, the working fluid area becomes "warm". On the contrary, when $R_{k}$ is very low (e.g. $R_{k}=0.1$, the porous zone will act as an adiabatic layer. Within the porous zone heat conduction plays a dominant role and the temperature gradient is steep. Accordingly, the fluid zone becomes very "cold" and its temperature approaches to the environmental temperature. The change of $R_{k}$ leads to significant difference in temperature distribution in the porous layer and in the fluid part of the cavity.

Figure 14 depicts the average Nusselt number at the hot wall. Compared with the thickness of the porous layer, the intensity of heat transfer is more sensitive to effective thermal conductivity of the porous layer, especially when $R_{k}$ is less 
than unity. If $R_{k}<1$, a slight increment of $R_{k}$ will cause a sharp fall of $N u$. While $R_{k}>5$, the decrease of $N u$ against $R_{k}$ increasing is extremely slight. Furthermore, $N u$ approaches to zero if $R_{k}$ is very high (e.g. $\left.R_{k}=10\right)$. Figure 15, in which the variation of local Nusselt number at the hot wall is plotted, can show more detailed information. Generally, the maximum heat transfer rate appears at the upper half part of the hot wall, except the scenarios where $R_{k} \gg 1$. For a small $R_{k}$ (e.g. $R_{k}=0.1$ ), the intensity of heat transfer is extremely non-uniform along the hot wall. The increasing of $R_{k}$ can erase such non-uniform as heat transfer becomes very weak on the hot wall for a high $R_{k}$. Consequently, it can be claimed that there is a critical $R_{k}$ which can completely alter the patterns of heat and mass transfer in the open-ended cavity. It is important for practical applications to determine their critical $R_{k}$ to guarantee the performance of systems. As highlighted by Fig.14, at the left side or at the right side of the critical point, the heat transfer efficiencies may be completely different and a slight offset may cause a considerable change.

Beckermann et al. $[16,17]$ investigated conjugate natural convection heat transfer in a closed cavity, in which a porous layer was placed. Their experimental and numerical data showed for a high effective-thermal-conductivity porous layer, natural convection in the porous layer was suppressed. The present study shows their conclusion is still valid for the open-ended counterpart. 


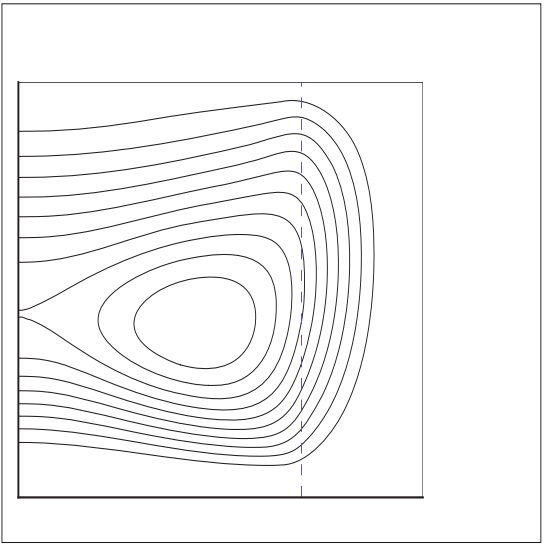

(a)

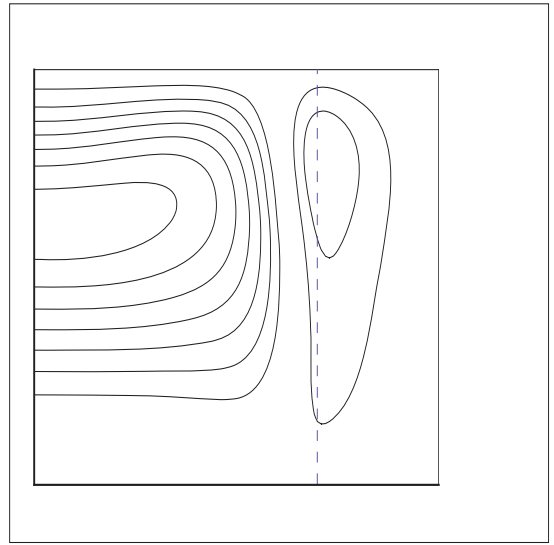

(b)

Fig. 12. Streamlines at (a) $R_{k}=0.1$ and (b) $R_{k}=10$.

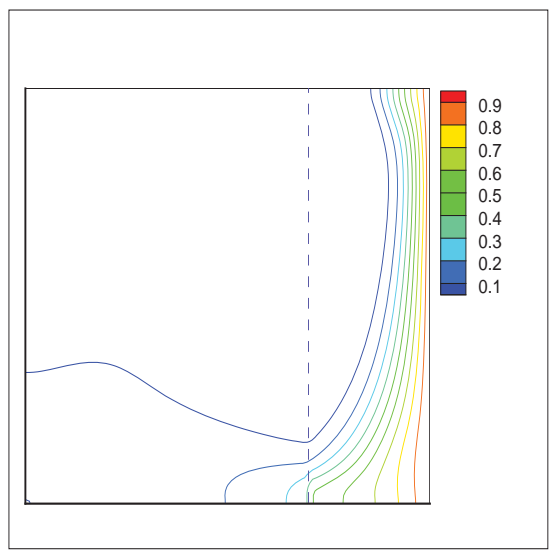

(a)

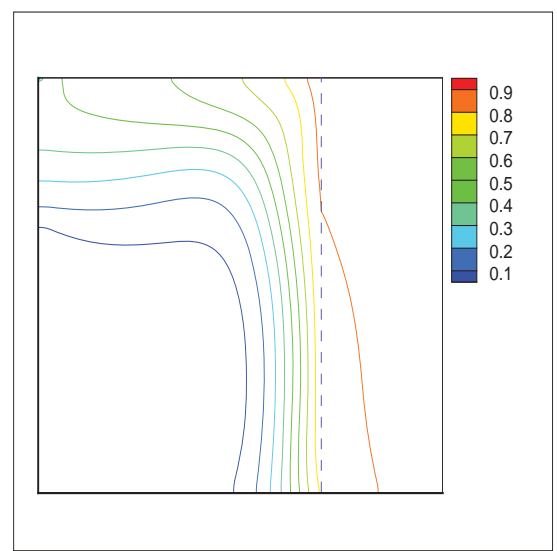

(b)

Fig. 13. Isotherms at at (a) $R_{k}=0.1$ and (b) $R_{k}=10$.

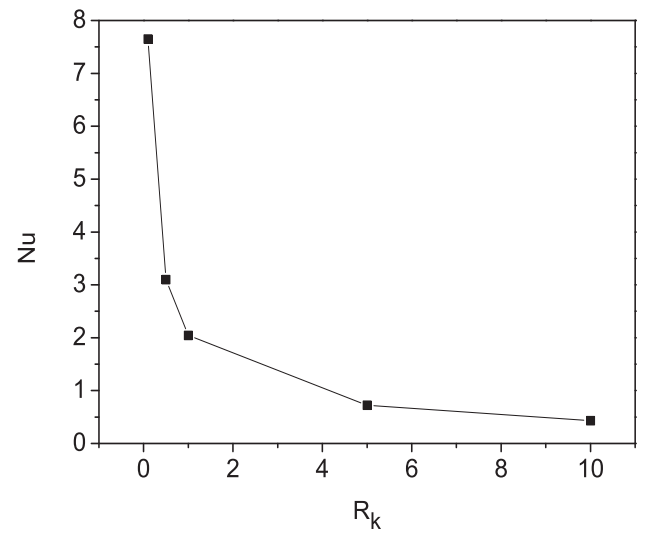

Fig. 14. Average Nusselt number at various $R_{k}$. 


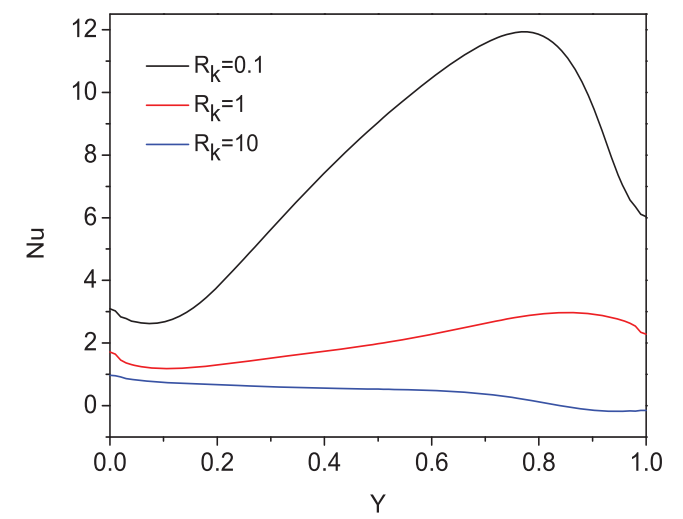

Fig. 15. Local Nusselt number along the hot wall at various $R_{k}$.

\subsection{Effect of permeability of porous layer}

In order to reveal the effect of permeability of the porous layer, we employ the Darcy number $D a$ as it represents the relative effect of the permeability of porous media. A larger $D a$ implies better permeability. In this subsection we set $\varepsilon=0.6, R a=10^{5}, \sigma_{\text {porous }}=1.0, d / L=0.3$ and $R_{k}=1$, while the Darcy number varies over a wide range $10^{-1} \leq D a \leq 10^{-5}$.

Figure 16 illustrates the streamline at various $D a$. It is not surprised that the flow pattern changes obviously with $D a$. When the permeability of the porous zone is high (e.g. $D a=10^{-1}$ ), the cold fluid from the environment can successfully penetrate the interface and form a large clockwise vortex. However, for a poor permeable porous layer (e.g. $D a=10^{-5}$ ), the cold fluid fails to do so and there emerge two vortices: a strong counterclockwise vortex near the open boundary and a weak clockwise one in the vicinity of the porous/fluid interface. In the fluid zone, the direction of flow circulation with a low $D a$ is 
completely reversed, as compared with its high permeable counterpart.

Figure 17 plots the corresponding isotherms. For a poor permeable porous layer (e.g. $D a=10^{-5}$ ), as the drag resistance is large and the saturating fluid moves slowly, heat conduction dominates the porous zone. With the increasing of permeability, the contribution of convective heat transfer ascends. Compared with that at $D a=10^{-5}$, as the direction of flow circulation at $D a=$ $10^{-1}$ is completed reversed, the temperature pattern is altered significantly versus the variation of $D a$. For example, when $D a=10^{-1}$, the lower half of the fluid zone is "warmer" than its upper half part. However, for $D a=10^{-5}$, the lower half of the fluid zone becomes "colder" than its upper half part.

The average Nusselt number at the hot wall is depicted by Fig.18. For the present investigated domain, one can observe that there is also a critical $D a$, which is located between $10^{-2}$ and $10^{-3}$. When $D a<10^{-3}$ or $D a>10^{-2}$, the variation of the average Nusselt number is slight. On the contrary, within the zone $10^{-3}<D a<10^{-2}$, a small offset of $D a$ will cause a great change of $N u$. Figure 19 presents the profile of local Nusselt number along the hot wall at various $D a$. It is clear heat transfer rate will become more non-uniform with a better permeable porous layer. For a good permeable porous layer (e.g. $D a \geq 10^{-3}$ ), the maximum of heat transfer rate appears at the upper half of the hot wall. However, for a porous layer with low permeability (e.g. $D a=10^{-5}$ ), the intensity of heat transfer will monotonically decrease against the height of the hot wall. 


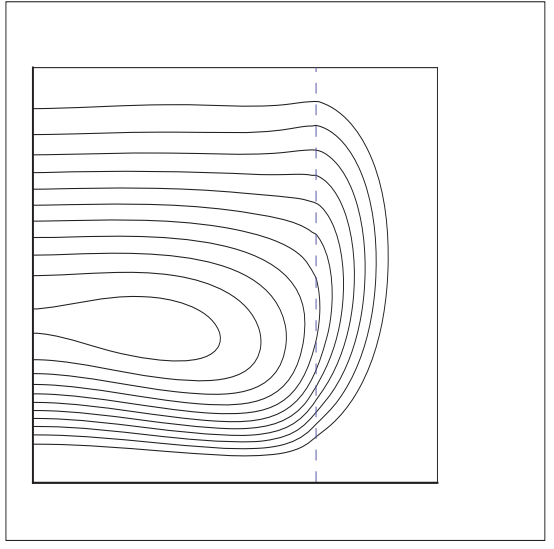

(a)

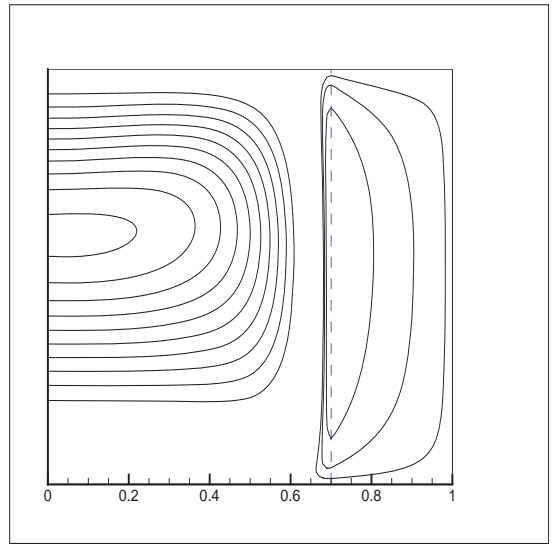

(b)

Fig. 16. Streamlines at (a) $D a=10^{-1}$ and (b) $D a=10^{-5}$.

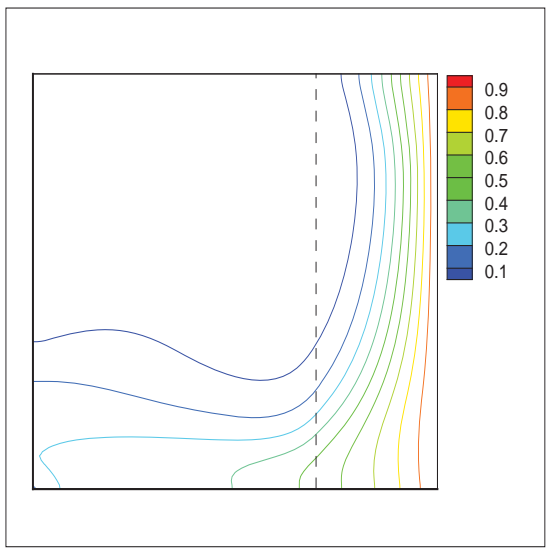

(a)

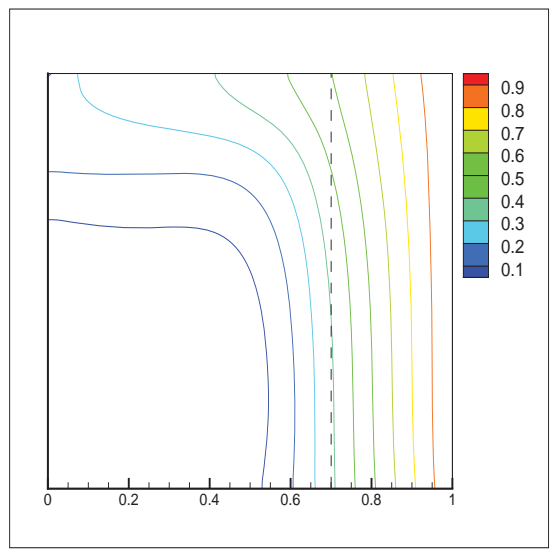

(b)

Fig. 17. Isotherms at at (a) $D a=10^{-1}$ and (b) $D a=10^{-5}$.

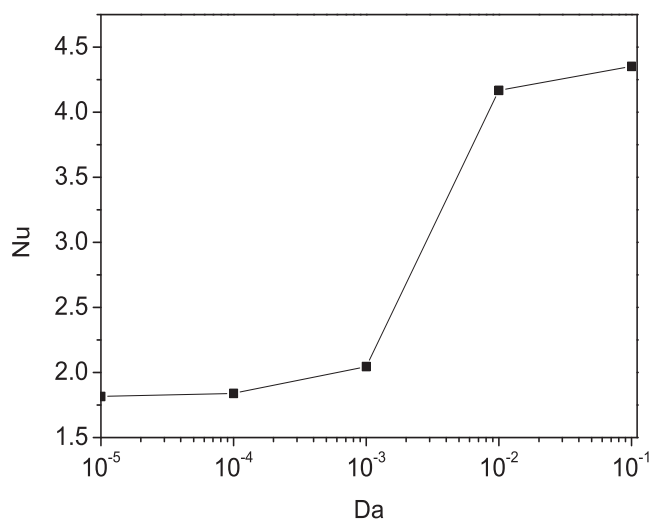

Fig. 18. Average Nusselt number at various $D a$. 


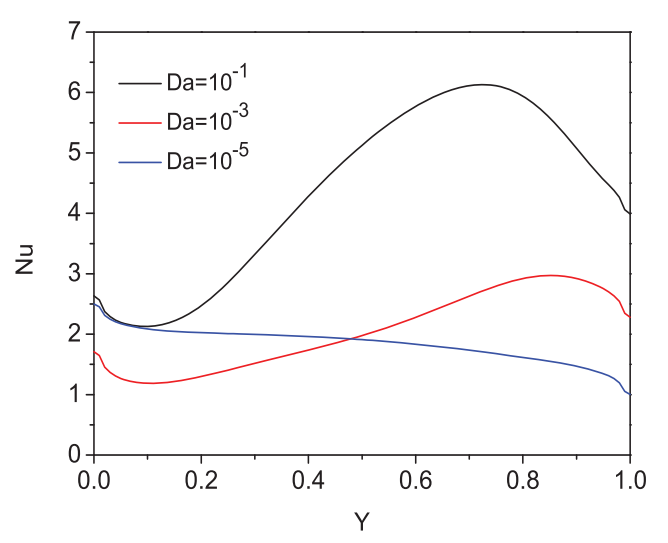

Fig. 19. Local Nusselt number along the hot wall at various $D a$.

\subsection{Effect of heterogeneous $\sigma$ on transient processes}

The purpose of this subsection is to show the applicability of the present model for modelling unsteady (or transient processes of) conjugate heat transfer on a fluid/porous interface. In the present study, heterogeneous $\sigma$ denotes $\sigma_{\text {porous }} \neq 1$, namely the heat capacitance across the porous/fluid interface is not homogeneous. According to Eqs. (3) and (6), one can find the choice of $\sigma_{\text {porous }}$ should not affect the final numerical results of a steady conjugate heat transfer problem. Figure 20 shows the isotherms at different $\sigma_{\text {porous }}$. The other parameters are $D a=10^{-3}, \varepsilon=0.6, R a=10^{5}, R_{k}=1$ and $d / L=0.3$. It is obvious the distributions of temperature at their steady status are completely identical, no matter whatever $\sigma_{\text {porous }}$ is. The present numerical results agree with the theoretical analysis. Unfortunately, if Guo's model [37] is adopted, we observe the computing will diverge quickly.

Figure 21 illustrates the profile of temperature along the fluid-porous inter- 


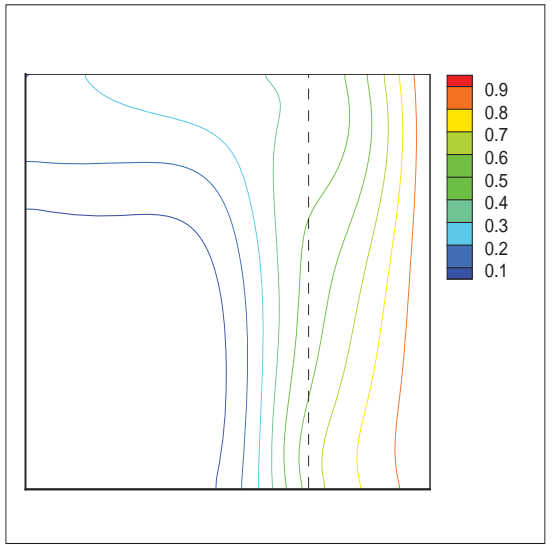

(a)

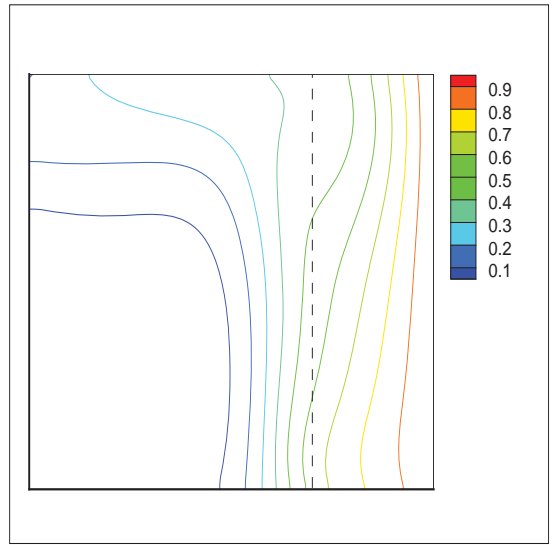

(b)

Fig. 20. Isotherms at (a) $\sigma_{\text {porous }}=0.5$ and (b) $\sigma_{\text {porous }}=5$.

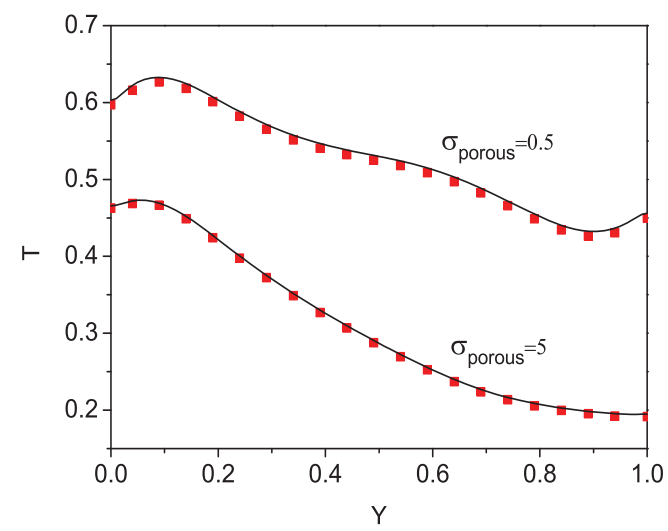

Fig. 21. Profile of temperature on fluid/porous interface at dimensionless time $t=10$ : solid line-present LB model, squares-SIMPLE algorithm used in [14].

face before the system achieves its steady status. One can observe that the effect of $\sigma_{\text {porous }}$ on the transient process is considerable. For a lower $\sigma_{\text {porous }}$, heat transfer is "quicker". Consequently, the system can achieve its steady status "faster". The present numerical results agree well with those obtained by the SIMPLE algorithm adopted in [14], which demonstrates the applicability of the present LB model for simulating unsteady (or transient) scenarios of conjugate heat transfer across a porous-fluid interface. 


\section{Conclusion}

Conjugate natural convection heat transfer in an open-ended square cavity, which is partially filled with porous media, is a useful research prototype to deepen our insight into many important practical applications, for example solar energy engineering and civil engineering. But surprising, until now there is no open literature on it. For traditional numerical approaches, it is a great challenge to model conjugate problems on fluid-porous interfaces. In the present work, firstly we develop a new LB approach to avoid complicated treatments in traditional numerical methods for conjugate problems. With the aid of this LB approach, we investigate the effects of thickness of porous layer, fluid-to-porous thermal conductivity ratio and permeability of porous layer on the characteristics of natural convection heat transfer in an open-ended porous-partially-filled square cavity. It is found that these factors all influence the patterns of flow field and temperature field significantly. Moreover, it is found that some conclusions, which were drew from a closed cavity partially filled by porous media, are still valid for its open-ended counterpart, although some are not. The present results may be useful for the relevant practical applications. For example, to get an optimized design of a solar thermal receiver partially filled by porous media [1], one must find out the critical value of thickness of porous layer and that of porous-to-fluid thermal conductivity ratio in advance, as a small offset from these critical values will cause the heat transfer performance to become poor. In addition, according to the present 
work, one must pay high attention to the effect of permeability of porous media on the uniform of temperature distribution in a solar thermal receiver, as seriously non-uniform temperature distribution will damage its life and overall efficiency. These conclusions can be obtained through the numerical experiments conducted in this paper and no open literature has shown us the information.

Finally, it has been demonstrated that the present model can be used for unsteady conjugate heat transfer problems, which will be considered in our future work.

\section{Acknowledgments}

S. Chen would acknowledge the support from the EPSRC project "UK Consortium on Mesoscale Engineering Sciences (UKCOMES)" (Grant no. EP/L00030X/1).

\section{References}

[1] Bairi A, Zarco-Pernia E, Maria JMG. A review on natural convection in enclosures for engineering applications. The particular case of the parallelogrammic diode cavity. Applied Thermal Engineering 2014;63: 304-322

[2] Mohamad AA. Natural convection in open cavities and slots. Numerical Heat Transfer, Part A: Applications 1995;27:705-716. 
[3] Bruce JM. Natural convection through openings and its application to cattle building ventilation. Journal of Agricultural Engineering Research 1978;23: 151167

[4] Montiel-Gonzalez M, Hinojosa JF, Villafan-Vidales HI, Bautista-Orozco A, Estrada CA. Theoretical and experimental study of natural convection with surface thermal radiation in a side open cavity. Applied Thermal Engineering $2015 ; 75: 1176-1186$

[5] Chehouani H, Said AAH, Fagrich ME. Heat transfer study of free convection through a horizontal open ended axisymmetric cavity using holographic interferometry. Experimental Thermal and Fluid Science 2015;60 308-316

[6] Hosseini R, Heyrani-Nobari MR, Hatam M. An experimental study of heat transfer in an open-ended vertical eccentric annulus with insulated and constant heat flux boundaries. Applied Thermal Engineering 2005;25: 1247-1257

[7] Mejri I, Mahmoudi A. MHD natural convection in a nanofluid-filled open enclosure with a sinusoidal boundary condition. Chemical Engineering Research and Design 2015;98: 1-16

[8] Saleem M, Hossain MA, Mahmud S, Pop I. Entropy generation in Marangoni convection flow of heated fluid in an open ended cavity. International Journal of Heat and Mass Transfer 2011; 54:4473-4484

[9] Haghshenas A, Nasr RM, Rahimian MH. Numerical simulation of natural convection in an open-ended square cavity filled with porous medium by lattice Boltzmann method. International Communications in Heat and Mass Transfer 2010;37: 1513-1519 
[10] Boetcher SKS, Sparrow EM. Buoyancy-induced flow in an open-ended cavity: Assessment of a similarity solution and of numerical simulation models. International Journal of Heat and Mass Transfer 2009;52: 3850-3856

[11] Mohamad AA, El-Ganaoui M, Bennacer R. Lattice Boltzmann simulation of natural convection in an open ended cavity. International Journal of Thermal Sciences 2009;48: 1870-1875

[12] Qin M, Belarbi R, Ait-Mokhtar A, Nilsson L. Coupled heat and moisture transfer in multi-layer building materials. Construction and Building Materials 2009;23: 967-975

[13] Wang W, Laumert B, Xu H, Strand T. Conjugate heat transfer analysis of an impinging receiver design for a dish-Brayton system. Solar Energy 2015;119: 298-309

[14] Bourouis A, Omara A, Abboudi S. Upward and downward conjugate mixed convection heat transfer in a partially porous cavity. International Journal of Numerical Methods for Heat \& Fluid Flow 2016;26:159-188.

[15] Chamkha AJ, Ismael MA. Conjugate heat transfer in a porous cavity filled with nanofluids and heated by a triangular thick wall. International Journal of Thermal Science 2013;67: 135-151.

[16] Beckermann C, Ramadhyani S, Viskanta R. Natural convection flow and heat transfer between a fluid layer and a porous layer inside a rectangular enclosure. Journal of Heat Transfer 1987;109: 363-370

[17] Beckermann C, Viskanta R, Ramadhyani S. Natural convection in vertical 
enclosures containing simultaneously fluid and porous layers. Journal of Fluid Mechanics 1988;186: 257-284

[18] Breton PL, Caltagirone JP, Arquis E. Natural Convection in a Square Cavity With Thin Porous Layers on Its Vertical Walls. Journal of Heat Transfer 1991;113: 892-898

[19] Singh AK, Leonardi E, Thorpe GR. Three-Dimensional Natural Convection in a Confined Fluid Overlying a Porous Layer. Journal of Heat Transfer 1993;115: $631-638$

[20] Nag A, Sarkar A, Sastri VMK. On the effect of porous thick horizontal partial partition attached to one of the active walls of a differentially heated square cavity. International Journal of Numerical Methods for Heat \& Fluid Flow $1994 ; 4: 399-411$.

[21] Song M, Viskanta R. Natural convection flow and heat transfer within a rectangular enclosure containing a vertical porous layer. International Journal of Heat and Mass Transfer 1994;37: 2425-2438

[22] Oosthuizen PH, Naylor D. Natural convective heat transfer from a cylinder in an enclosure partly filled with a porous medium. International Journal of Numerical Methods for Heat \& Fluid Flow 1996; 6:51-63

[23] Al-NimrA MA, Khadrawi F. Transient Free Convection Fluid Flow in Domains Partially Filled with Porous Media. Transport in Porous Media 2003; 51:157-172

[24] Arpino F, Massarotti N, Mauro A. Efficient three-dimensional FEM based algorithm for the solution of convection in partly porous domains. International 
Journal of Heat and Mass Transfer 2011;54:4495-4506

[25] Sheremet MA, Trifonova TA. Unsteady Conjugate Natural Convection in a Vertical Cylinder Partially Filled with a Porous Medium. Numerical Heat Transfer, Part A: Applications 2013; 64: 994-1015

[26] Mondal PK, Dholey S. Effect of conjugate heat transfer on the irreversibility generation rate in a combined CouetteePoiseuille flow between asymmetrically heated parallel plates: The entropy minimization analysis. Energy 2015;83: 5564

[27] Uddin MJ, Beg OA, Uddin MN. Energy conversion under conjugate conduction, magneto-convection, diffusion and nonlinear radiation over a non-linearly stretching sheet with slip and multiple convective boundary conditions. Energy 2016;115:1119-1129

[28] Succi S. The lattice Boltzmann equation for fluid dynamics and beyond. Oxford: Oxford university press;2001.

[29] Karani H, Huber C. Lattice Boltzmann formulation for conjugate heat transfer in heterogeneous media. Physical Review E 2015;91:023304/1-023304/10.

[30] Hu Y, Li D, Shu S, Niu X. Full Eulerian lattice Boltzmann model for conjugate heat transfer. Physical Review E 2015;92:063305/1-063305/12

[31] Le G, Oulaid O, Zhang JF. Counter-extrapolation method for conjugate interfaces in computational heat and mass transfer. Physical Review E 2015;91:033306/1-033306/11

[32] Chen S, Yan YY, Gong W. A simple lattice Boltzmann model for conjugate heat 
transfer research. International Journal of Heat and Mass Transfer 2017;107: $862-870$

[33] Pareschi G, Frapolli N, Chikatamarla SS, Karlin IV. Conjugate heat transfer with the entropic lattice Boltzmann method. Physical Review E 2016;94:013305/1-013305/16

[34] Lu JH, Lei HY, Dai CS. A simple difference method for lattice Boltzmann algorithm to simulate conjugate heat transfer. International Journal of Heat and Mass Transfer 2017;114: 268-276

[35] Gao D, Chen Z, Chen L, Zhang D. A modified lattice Boltzmann model for conjugate heat transfer in porous media. International Journal of Heat and Mass Transfer 2017;105: 673-683

[36] Chen S. Simulation of conjugate heat transfer between fluid-saturated porous media and solid wall. International Journal of Thermal Sciences 2018;124: 477483

[37] Guo Z, Zhao TS. A lattice Boltzmann model for convection heat transfer in porous media. Numerical Heat Transfer, Part B 2005; 47: 157-177

[38] Chen S, Yang B, Zheng C. A lattice Boltzmann model for heat transfer in heterogeneous media. International Journal of Heat and Mass Transfer 2016; 102: $637-644$

[39] Chen S, Yang B, Zheng C. A lattice Boltzmann model for heat transfer in porous media. International Journal of Heat and Mass Transfer 2017;111: 1019-1022

[40] Chen S, Liu Z, Zhang C, He Z, Tian Z, Shi B, Zheng C. A novel coupled 
lattice Boltzmann model for low Mach number combustion simulation. Applied Mathematics and Computation 2007;193 : 266-284. 


\section{Nomenclature}

$u_{\alpha}, u_{\beta}$ fluid velocity $(\mathrm{m} / \mathrm{s})$

$p$ fluid pressure $\left(N / m^{2}\right)$

$T$ temperature $(K)$

$g_{\alpha}$ gravitational acceleration $\left(\mathrm{m} / \mathrm{s}^{2}\right)$

$R_{k}$ porous-to-fluid thermal conductivity ratio

$g_{j}$ pseudo-particle distribution

$t$ time $(s)$

$c$ pseudo-particle speed

$e_{j}$ discrete velocity direction of pseudo-particle

$n_{\alpha}$ normal direction

$R a$ Rayleigh number

$N u$ Nusselt number

Da Darcy number

$L$ height or length of cavity $(m)$

$d$ thickness of porous media $(m)$ 
$C$ heat capacitance, namely the product of density and specific heat capacity $\left(k g^{2} / K s^{2} m\right)$

Greek symbols

$\varepsilon$ porosity of porous media

$\nu_{e}$ effective kinematic viscosity $\left(\mathrm{m}^{2} / \mathrm{s}\right)$

$\kappa_{e}$ effective thermal diffusivity $\left(\mathrm{m}^{2} / \mathrm{s}\right)$

$\sigma$ porous to fluid heat capacitance ratio

$\beta$ thermal expansion coefficient $(1 / K)$

$\tau_{T}$ dimensionless relaxation time

$\omega_{j}$ weight coefficient

$\epsilon$ Knudsen number

$\delta_{\alpha \beta}$ kronecker delta

Subscripts and superscripts

$f$ fluid

porous porous media

0 reference value 
$e q$ equilibrium status 